\title{
DISK-LIKE FUNCTIONS
}

\author{
RICHARD J. LIBERA *
}

(Received 4 November 1968)

Communicated by E. Strzelecki

\section{Introduction}

The class $\mathscr{S}$ of functions $f(z)$ which are regular and univalent in the open unit disk $\Delta=\{z:|z|<1\}$ each normalized by the conditions

$$
f(0)=0 \text { and } f^{\prime}(0)=1
$$

has been studied intensively for over fifty years. A large and very successful portion of this work has dealt with subclasses of $\mathscr{S}$ characterized by some geometric property of $f[\Delta]$, the image of $\Delta$ under $f(z)$, which is expressible in analytic terms. The class of starlike functions in $\mathscr{S}$ is one of these [3]; $f(z)$ is starlike with respect to the origin if the segment $[0, f(z)]$ is in $f[\Delta]$ for every $z$ in $\Delta$ and this condition is equivalent to requiring that

have a positive real part in $\Delta$.

$$
z f^{\prime}(z) / f(z)
$$

In this note the class of 'disk-like' functions is introduced by placing restrictions on the behavior of the imaginary part of (1.2) and a representation formula for these functions is given in terms of Robertson's functions which are starlike in one direction [4].

\section{The class $\mathscr{D}$}

In the definition which follows the notation $g(t) \in \uparrow(a, b)$ means that $g(t)$ is strictly increasing in the interval $a<t<b . g(t) \in \downarrow(a, b)$ has a similar meaning.

Definition 1: $f(z)$ is regular in $\Delta$, satisfies $(1.1)$ and $f(z) \neq 0$ for $z$ in $\Delta$ unless $z=0 . f(z)$ is disk like with respect to the origin in $\Delta$, or $f(z) \in \mathscr{D}$, if and only if one of the following conditions is satisfied:

i) There exists a constant $\rho=\rho(f(z))>0$ and two functions $\theta_{k}(r)=$ $\theta_{k}(r ; f(z)), k=1,2 ; 0<\theta_{2}(r)-\theta_{1}(r)<2 \pi$; such that for $\rho<r<1$

$$
\left|f\left(r e^{i \theta}\right)\right| \in \downarrow\left(\theta_{1}(r), \theta_{2}(r)\right) \text { and }\left|f\left(r e^{i \theta}\right)\right| \in \uparrow\left(\theta_{2}(r), \theta_{1}(r)+2 \pi\right) .
$$

* This work was supported by National Science Foundation Grant NSF-GP-7439. 
ii) $f(z)$ is regular in $\bar{\Lambda}$, the closure of $\Delta$, and there exist real numbers $\theta_{1}$ and $\theta_{2}, 0<\theta_{2}-\theta_{1}<2 \pi$, such that

$$
\left|f\left(e^{i \theta}\right)\right| \in \downarrow\left(\theta_{1}, \theta_{2}\right) \text { and }\left|f\left(e^{i \theta}\right)\right| \in \uparrow\left(\theta_{2}, \theta_{1}+2 \pi\right) .
$$

Functions in $\mathscr{D}$ are not necessarily univalent; $\mathscr{D}^{\prime}$ will denote the class of univalent members of $\mathscr{D}$, i.e., $\mathscr{D}^{\prime}=\mathscr{D} \cap \mathscr{S} . \theta_{1}(r)$ may be chosen so that $0 \leqq \theta_{1}(r)$ $<2 \pi$ and a similar choice can be made for $\theta_{1}$ in (ii). Hereafter, unless otherwise implied, $f(z)$ in $\mathscr{D}$ will satisfy part (i) of the definition as this is no restriction on the development.

A subclass of $\mathscr{D}$, the circularly symmetric functions, was introduced earlier by Jenkins [1] and has appeared in the recent investigations of Krzyż and Reade [2]. Tammi [5] has obtained distortion theorems and coefficient bounds for functions defined in terms of restrictions on the quotient (1.2).

A geometric interpretation of the conditions of Definition 1 may be given. Let $f(z), \rho, \theta_{1}(r)$ and $\theta_{2}(r)$ satisfy (i), let $\left|f\left(r e^{i \theta_{1}(r)}\right)\right|=R_{1}(r)$ and $\left|f\left(r e^{i \theta_{2}(r)}\right)\right|=$ $R_{2}(r)$ and let $C_{r}$, the image of $|z|=r$ under $f(z)$, enclose a domain $D_{r}$. Then $C_{r}$ is contained entirely in the annulus $R_{2}(r) \leqq|w| \leqq R_{1}(r)$ and $C_{r}$ intersects every circle $|w|=R, R_{2}(r)<R<R_{1}(r)$, exactly twice (perhaps in the same point) for $\rho<r<1$. If for $R_{2}(r)<R<R_{1}(r)$ we let $\Phi_{1}(r ; R)$ and $\Phi_{2}(r ; R)$ be the arguments, chosen to be unique by continuity, of the intersection of $|w|=R$ with the arcs $\left\{f\left(r e^{i \theta}\right) \mid \theta_{1}(r)<\theta<\theta_{2}(r)\right\}$ and $\left\{f\left(r e^{i \theta}\right) \mid \theta_{2}(r)<\theta<\theta_{1}(r)+2 \pi\right\}$, respectively, then $D_{r}$ contains the arc $\operatorname{Re}^{i \Phi}, \Phi_{2}(r ; R)<\Phi<\Phi_{1}(r ; R)$. It is clear that $f(z)$ is not univalent in $\Delta$ if there exist $r$ and $R$ such that $\Phi_{1}(r ; R)-\Phi_{2}(r, R) \geqq 2 \pi$ for all choices of arguments. On the other hand $f(z)$ is univalent in $\Delta$ whenever $0<\Phi_{1}(r ; R)-\Phi_{2}(r ; R)<2 \pi$ for all admissible $r$ and $R$ and appropriate $\Phi_{1}(r ; R)$ and $\Phi_{2}(r ; R)$, and conversely; hence $f(z) \in \mathscr{D}^{\prime}$, or $f(z)=z$, if and only if every circle centered at the origin meets $f[\Delta]$ in a single, non-overlapping arc or not at all. This gives rise to the following observation which we will use: $f(z)$ is univalent for $|z| \leqq r$ if the plane can be cut from $f\left(r e^{i \theta_{2}(r)}\right)$ to $\infty$ by a curve which does not meet $C_{r}$ in any point other than $f\left(r e^{i \theta_{2}(r)}\right)$.

Returning now to the definition, we see that for $\rho<r<1,(2.1)$ can be written as

$$
\begin{aligned}
\frac{\partial}{\partial \theta}\left|f\left(r e^{i \theta}\right)\right|<0 & \text { for } \theta_{1}(r)<\theta<\theta_{2}(r) \\
>0 & \text { for } \theta_{2}(r)<\theta<\theta_{1}(r)+2 \pi
\end{aligned}
$$

or as

Because

$$
\begin{array}{ll}
\frac{\partial}{\partial \theta} \log \left|f\left(r e^{i \theta}\right)\right|<0 & \text { for } \theta_{1}(r)<\theta<\theta_{2}(r) \\
>0 & \text { for } \theta_{2}(r)<\theta<\theta_{1}(r)+2 \pi .
\end{array}
$$

$$
\operatorname{Re}\left\{\frac{\partial}{\partial \theta} \log f(z)\right\}=\operatorname{Re}\left\{\frac{d}{d z} \log f(z) \cdot \frac{d z}{d \theta}\right\}=\operatorname{Re}\left\{i \frac{z f^{\prime}(z)}{f(z)}\right\}=-\operatorname{Im}\left\{\frac{z f^{\prime}(z)}{f(z)}\right\}
$$

for $z=r e^{i \theta},(2.2)$ is equivalent to 


$$
\operatorname{Im}\left\{\frac{r e^{i \theta} f^{\prime}\left(r e^{i \theta}\right)}{f\left(r e^{i \theta}\right)}\right\} \begin{array}{ll}
>0 & \text { if } \theta_{1}(r)<\theta<\theta_{2}(r), \\
<0 & \text { if } \theta_{2}(r)<\theta<\theta_{1}(r)+2 \pi .
\end{array}
$$

The last form upon normalization relates to functions starlike in one direction. Letting

$$
f(z)=z+\sum_{k=2}^{\infty} a_{k} z^{k}, z \in \Delta,
$$

then

$$
\frac{z f^{\prime}(z)}{f(z)}-1=a_{2} z-\left(2 a_{3}-a_{2}^{2}\right) z^{2}+\cdots
$$

Consequently $a_{2} \neq 0$, otherwise (2.7) has a multiple zero which is a contradiction of (2.5). It is, therefore, no restriction to assume that $a_{2}$ is real and positive. The above discussion yields the following conclusions.

THEOREM 1: $f(z)$ has the form (2.6) and $a_{2}>0 . f(z)$ is in $\mathscr{D}$ if and only if

$$
a_{2}^{-1}\left\{\frac{z f^{\prime}(z)}{f(z)}-1\right\}
$$

is starlike in the direction of the real axis.

It should be noticed that if $a_{2} \neq 0$, then $f(z)$ is not odd; this is consistent with the geometrical interpretation given above. Consequently the identity function $f(z)=z$ is not in $\mathscr{D}$. A modification of Definition 1 to admit simple monotonic rather than strictly monotonic functions in (2.1) and (2.2) admits the identity function into $\mathscr{D}$, in which case (2.7) is identically zero.

Making use of the fact that $g(z)$ regular in $\Delta$ and normalized by $(1.1)$ is convex in one direction if and only if $z g^{\prime}(z)$ is starlike in one direction [4] we can write the last theorem in another form.

CoROllary: $f(z)=z+\sum_{k=2}^{\infty} a_{k} z^{k}, a_{2}>0$, is in $\mathscr{D}$ if and only if $f(z)=z e^{a_{2} g(z)}$ and $g(z)$ is convex in the direction of the imaginary axis.

This can be obtained directly from Theorem 1 or by observing that for $z=r e^{i \theta}, \rho<r<1,(2.4)$ is equivalent to

$$
\begin{aligned}
\operatorname{Re} \frac{\partial}{\partial \theta} \log \left(\frac{f(z)}{z}\right)<0 & \text { for } \theta_{1}(r)<\theta<\theta_{2}(r) \\
>0 & \text { for } \theta_{2}(r)<\theta<\theta_{1}(r)+2 \pi
\end{aligned}
$$

Hence,

$$
\log \left(\frac{f(z)}{z}\right)=a_{2} z+\left(a_{3}-\frac{a_{2}^{2}}{2}\right) z^{2}+\cdots
$$

is convex in one direction. 


\section{A univalent subclass of $\mathscr{D}$}

For a fixed $\phi,-\pi<\phi \leqq \pi$, we say that $f(z)$ regular in $\Delta$, or in $\bar{\Delta}$, and normalized by (1.1) is in $\mathscr{R}_{\phi}$ if and only if $f(z)$ is starlike in the direction with inclination $\phi$. That is, the line $w=t e^{i \phi}, t$ real, intersects $C_{r}$, the image of $|z|=r$ under $f(z)$, for $r$ near or equal to 1 exactly twice. Evidently $f(z) \in \mathscr{R}_{\phi}$ whenever $e^{-i \phi} f\left(z e^{i \phi}\right)$ is starlike in the direction of the real axis, and conversely. Consequently, there exist functions $\tau_{1}(r ; f(z))$ and $\tau_{2}(r ; f(z))$ such that for $\sigma<r<1$, or for $r=1$, and suitable choice of arguments, $\phi<\arg \left\{f\left(r e^{i \phi}\right)\right\}<\phi+\pi$ whenever

and

$$
\tau_{1}(r ; f(z))<\theta<\tau_{2}(r ; f(z))
$$

whenever

$$
\phi+\pi<\arg \left\{f\left(r e^{i \phi}\right)\right\}<\phi+2 \pi
$$

$$
\tau_{2}(r ; f(z))<\theta<\tau_{1}(r ; f(z))+2 \pi .
$$

Clearly $\sigma$ depends on $f(z), \sigma=\sigma(f(z))$, and in the case $r=1$ it is assumed that $f(z)$ is holomorphic in $\bar{\Delta}$.

Using these ideas we may restate Theorem 1 in the following useful form.

THEOREM 2: $f(z)$ has series representation (2.6) and $a_{2} \neq 0 . f(z)$ is in $\mathscr{D}$ if and only if

for $\phi=\operatorname{Arg}\left\{a_{2}^{-1}\right\}$.

$$
a_{2}^{-1}\left\{\frac{z f^{\prime}(z)}{f(z)}-1\right\} \in \mathscr{R}_{\phi}
$$

Choose $\alpha=\operatorname{Arg} a_{2}$, where Arg denotes the principal argument, then

$$
f_{0}(z)=e^{i \alpha} f\left(z e^{-i \alpha}\right)=z+\left|a_{2}\right| z^{2}+\cdots
$$

is in $\mathscr{D}$, since membership in $\mathscr{D}$ is preserved under rotation. Therefore writing

$$
\left|a_{2}\right|^{-1}\left\{\frac{z f_{0}^{\prime}(z)}{f_{0}(z)}-1\right\}=g(z)
$$

where $g(z)$ is starlike in the direction of the real axis, yields, upon substitution, the relation

$$
a_{2}^{-1}\left\{\frac{z f^{\prime}(z)}{f(z)}-1\right\}=e^{-i \alpha} g\left(z e^{i \alpha}\right)
$$

The last function is in $\mathscr{R}_{-\alpha}$ and $-\alpha=\phi$.

DEFINITION 2: $f(z)$ and $g(z)$, both in $\mathscr{R}_{\phi}$, are similar if and only if

$$
\tau_{k}(r ; f(z))=\tau_{k}(r ; g(z)), k=1,2
$$

for $r=1$ when $f(z)$ and $g(z)$ are regular in $\bar{\Delta}$ and for $\sigma<r<1$, 
otherwise.

$$
\sigma=\sigma(f(z) ; g(z))
$$

Let $\tau_{k}(r)=\tau_{k}(r ; f(z))=\tau_{k}(r ; g(z)), k=1,2$, then a geometric interpretation of similarity is that for $r$ near or equal to $1, f\left(r e^{i \tau_{1}(r)}\right)$ and $g\left(r e^{i \tau_{1}(r)}\right)$ both lie on one ray of the line $w=t e^{i \phi}, t$ real, whereas $f\left(r e^{i \tau_{2}(r)}\right)$ and $g\left(r e^{i \tau_{2}(r)}\right)$ both lie on the complementary ray. $\mathscr{R}_{0}$ is the class of functions starlike in the direction of the real axis and any two typically-real functions, all of which are in $\mathscr{R}_{0}$, are similar.

THEOREM 3: If

$$
f(z)=z+\sum_{k=2}^{\infty} a_{k} z^{k}, a_{2} \neq 0, \text { and } g(z)=a_{2}^{-1}\left\{\frac{z f^{\prime}(z)}{f(z)}-1\right\}
$$

are similar and in $\mathscr{R}_{\phi}, \phi=\operatorname{Arg}\left\{a_{2}^{-1}\right\}$, then $f(z)$ is univalent in $\Delta$.

To give a proof let $\tau_{k}(r), k=1,2$, and $\sigma$ be as in Definition 2 and the above paragraph. Suppose furthermore that $f\left(r e^{i \tau_{1}(r)}\right)$ and $g\left(r e^{i \tau_{1}(r)}\right)$ fall on the ray $w=t e^{i \phi}, t>0$, for $\sigma<r<1$. Then for $z=r e^{i \phi}, \sigma<r<1$ and appropriate choice of arguments

and

$$
\phi<\arg \{g(z)\}<\phi+\pi \text { for } \tau_{1}(r)<\theta<\tau_{2}(r)
$$

$$
\phi+\pi<\arg \{g(z)\}<\phi+2 \pi \text { for } \tau_{2}(r)<\theta<\tau_{1}(r)+2 \pi
$$

or

and

$$
0<\arg \left\{e^{-i \phi} g(z)\right\}<\pi \text { when } \tau_{1}(r)<\theta<\tau_{2}(r)
$$

$$
\pi<\arg \left\{e^{-i \phi} g(z)\right\}<2 \pi \text { when } \tau_{2}(r)<\theta<\tau_{1}(r)+2 \pi .
$$

This is equivalent to

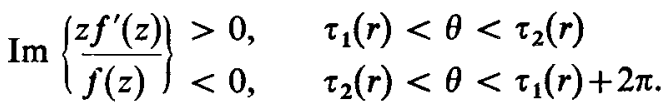

Consequently, $\left|f\left(r e^{i \phi}\right)\right|$ is strictly decreasing for $\tau_{1}(r)<\theta<\tau_{2}(r)$ and for a fixed $r, \rho<r<1$, hence $f\left(r e^{i \phi}\right)$ cuts every circle $|w|=R,\left|f\left(r e^{i \tau_{2}(r)}\right)\right|<R<\left|f\left(r e^{i \tau_{1}(r)}\right)\right|$ exactly once at a point $\operatorname{Re}^{i \mu}, \phi<\mu<\phi+\pi$ because $f(z) \in \mathscr{R}_{\phi}$. We see in the same way that every semicircle $\operatorname{Re}^{i \mu}, \phi+\pi<\mu<\phi+2 \pi$ is intercepted only once. Therefore $f(z)$ is univalent on $|z|=r$ and is, for that reason [3], univalent for $|z| \leqq r$. A similar argument covers the remaining cases.

\section{References}

[1] J. A. Jenkins, 'On circularly symmetric functions', Proc. Amer. Math. Soc., 6 (1955), 620-624.

[2] J. Krzyż and M. O. Reade, 'Koebe domains for certain classes of analytic functions', J. d'Analyse Math., 18 (1967), 185-195. 
[3] Z. Nehari, Conformal Mapping, (McGraw-Hill, New York, 1952).

[4] M. S. Robertson, 'Analytic functions starlike in one direction', Amer. J. Math., 58 (1936), $465-472$.

[5] O. Tammi, 'On certain combinations of the coefficients of schlicht functions', Ann. Acad. Sci. Fennicae Ser. A. I. Máth.-Phys, No. 140, (1952) 13 pp.,

The University of Delaware

Newark, Delaware 